\title{
THE PERCEIVED VALUE OF THE IV TEAM ASSISTANCE, MANAGEMENT AND EDUCATIONAL ACTIVITY: Results of a hospitalist survery
}

\author{
Montealegre Sanz, M. PhD; Cubero Pérez MA. RN; Gallardo Garrido P. RN; Varga \\ Vázquez S. RN; González Martín J . \\ IV TEAM. Hospital Clinico San Carlos. Madrid. Spain
}

\section{Introdution}

Since the Intravenous Therapy Team (IV Team) creation the use of peripherally inserted central catheters (PICCs) and Midline are growing and its use has been incorporated in new clinical areas.

This expanding in its use is associated with a higher rate of complications like thrombosis or infection. Moreover, some PICCs may not be placed for clinically valid reasons.

IV Team results key for defining appropriate indications for catheter insertion and maintenance as major endpoints for patient safety.

\section{Method}

We elaborated a survey addressed to hospitalization nurses attending patients with Midline or PICCs.

Survey evaluated 12 items related to IV Team procedures, material resources and algorithms available for management of catheter-related complications.

Satisfaction survey of the intravenous therapy team for nurses:

1) Do you know the name of the professional IV TEAM who inserts PICC and midline?

2) Does IV team inform you about inserted catheters and care of them?

3) Before the insertion midline/PICC, does IV team review the treatment,

coagulation profile and personal history of patients?

4) Do you consider suitable that IV team do the monitoring and dressing change of the catheters?

5) Would you like to do the monitoring and dressing change of the catheters?

6) Do you find useful to have an algorithm in case of possible complications?

Thrombosis, catheter-related bloodstream infection, obstruction and migration

7) Point out which algorithm consider most helpful: phlebitis, thrombosis,

catheter-related bloodstream infection, obstruction or migration.

8) Would you like to receive a specific patient-card for register the PICC/midline?

9) When a post-implantation complication appears, does the IV team solve it?

10) Do you think that exist a long waiting list for catheter insertion?

11) In case of vascular depletion, do you consider the midline catheter useful for the patient improvement?

12) What value do you give to the midline catheter alternative?

\section{Results}

115 nurses from 18 different units of the hospital completed the questionnaire. Professionals surveyed felt very confident with the IV Team

$78 \%$ of nurses consider IV Team monitorization and dressing change of the catheters as optimum care during hospitalization period, whereas the rest of them suggest a direct management by the head nurse of each medical unit.

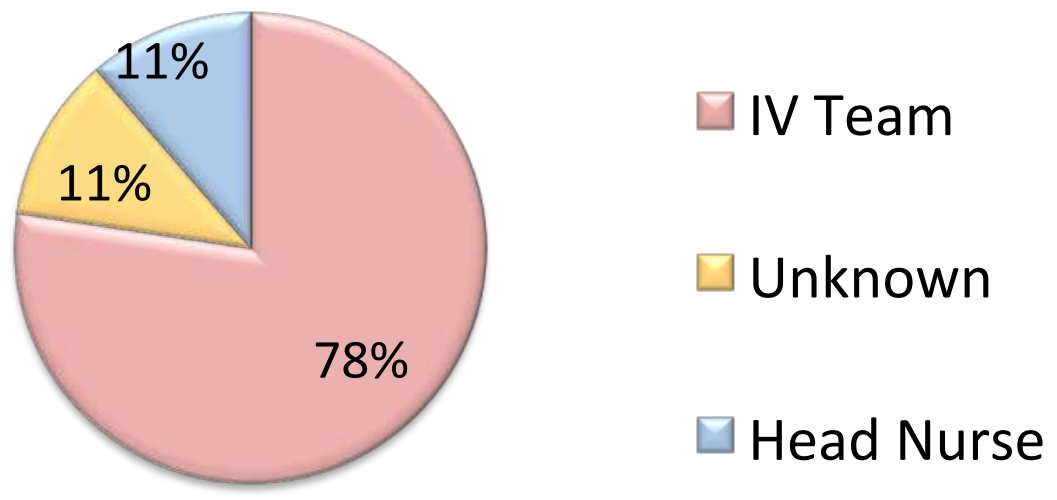

Algorithms for complications were appreciated like very useful for the $85 \%$ of respondents, resulting as the most valuated the central line associated bloodstream infection protocol.

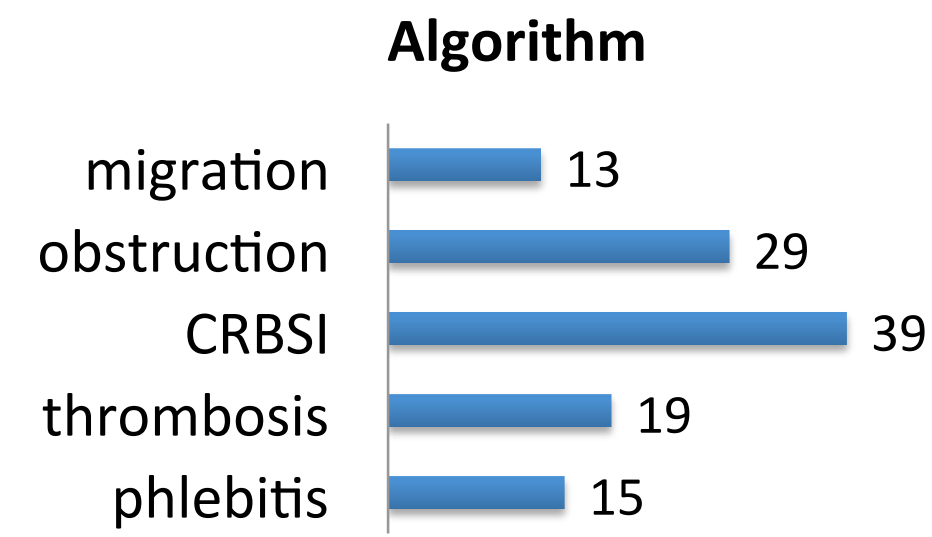

They highlighted the Midline as an especially useful device in poor peripheral venous access, considering it like a very interesting alternative to PICC or other central venous catheters.

1 (Absolutely disagree)

(Absolutely agree) 10

\section{Discussion and Conclusions}

- IV Team engagement with all the rest of clinical units in the hospital is increasingly widest and its contribution in the safety of patients with catheters is fully recognized.

- Nurses attending this population demand the implication of the IV Team in the daily management of these devices, especially after the implantation for the attention of possible subsequent complications.

- The selection of appropriate catheter should take in account patients or venous access characteristics and nature, duration and properties of the infusate.

- Algorithms for management of catheter-related complications has been adequately validated and implemented by the scientific community. The perception of its usefulness in the real world daily practice as a critical tool to preventing costly and potentially fatal adverse events has been endorsed in our project.

\section{References}

